\title{
Design and Synthesis of Amide Derivatives as $S$-Adenosyl- L-Homocysteine Hydrolase Inhibitors
}

\author{
Xiangduan Tan, ${ }^{a}$ Panfeng Wang, ${ }^{b}$ Siyun Nian, ${ }^{a}$ and Guoping Wang*,a,b \\ ${ }^{a}$ Shanghai Institute of Pharmaceutical Industry, China State Institute of Pharmaceutical Industry; Shanghai 200437, \\ China: and ${ }^{b}$ Shanghai Shyndec Pharmaceutical Co., Ltd.; Shanghai 200437, China. \\ Received August 7, 2013; accepted October 20, 2013; advance publication released online October 25, 2013
}

In this study, a series of amide derivatives were synthesized and evaluated for their $S$-adenosyl-Lhomocysteine hydrolase (SAHase) inhibitory activities. The results demonstrated that most of compounds displayed potent SAHase inhibitory activities. Interestingly, compounds 11 and 14 exhibited more potent inhibitory effects than the aristeromycin, one of the most potent SAHase inhibitors known so far. Compounds $12,13,15$ and 17 exhibited a moderate effect $\left(\mathrm{IC}_{50}<10.0 \mu \mathrm{M}\right)$. The structure-activity relationship found that compounds with substituted indazole-5-yl group at Ar position and ethylamino group at the side chain showed better SAHase inhibitory activities.

Key words $S$-adenosyl-L-homocysteine hydrolase; $S$-adenosylhomocysteine; SAHase inhibitor; homocysteine; amide derivative

$S$-Adenosyl-L-homocysteine hydrolase (SAHase) can catalyze the reversible hydrolysis of $S$-adenosylhomocysteine (SAH) to adenosine (ADO) and L-homocysteine (HCY). ${ }^{1)}$ Transmethylation reactions are involved in various biological phenomena related to the development of pathological processes. ${ }^{2)} S$-Adenosyl-L-methionine (AdoMet) seems to be the most versatile methyl donor in mammalian systems. The formation of SAH from AdoMet, and the inhibition of cellular SAHase results in an intracellular accumulation of $\mathrm{SAH}$, which leads to feedback inhibition of AdoMet dependent methylations. ${ }^{3)}$

In recent years, SAHase has become an attractive target for drug design, SAHase inhibitors have been shown to exhibit antiviral, ${ }^{4,5)}$ antiparasitic, ${ }^{6}$ anti-cancer, ${ }^{7)}$ and immunosuppressive effect, ${ }^{8,9)}$ SAHase inhibitors have also been shown to exhibit plasma homocysteine-lowering effect. ${ }^{10,11)}$ Numerous SAHase inhibitors have been extensively reported in literature, and all the existing SAHase inhibitors can be divided into three types according to the mechanisms of enzyme inhibition. Most of SAHase inhibitors were type I or type II inhibitor which were irreversible inhibitors, ${ }^{10)}$ including aristeromycin, neplanocin A and 3-deazaadenosine (3-DZA) (Fig. 1). Type III inhibitors can reversibly bind to the open form of the enzyme, maintaining a similar potency with much reduced toxicity, such as DZ2002 ${ }^{8,9)}$ (Fig. 1). However, most of the existing SAHase inhibitors are adenosine analogues, and most of the work previously reported on inhibitors of this enzyme has focused on systematically altering the structure of adenosine

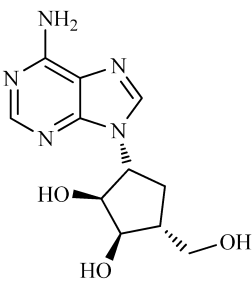

Aristeromycin

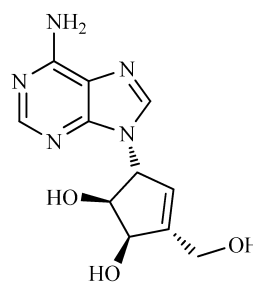

Neplanocin A and evaluating the derivatives. ${ }^{12)}$ There have been very few studies on new structure as SAHase inhibitors.

Recent studies found the $N$-(carbamoylmethyl)glycinamide derivatives exhibited SAHase inhibitory activities. ${ }^{13)}$ In terms of the structure of these compounds, we chose the different substituted phenylazanediyl diacetic acid derivatives as the core scaffold, which firstly reacted with $N$-methylisoindolin2-amine hydrochloride to obtain monoacid derivatives, and then acylation reaction with ethylenediamine derivatives to configure the side chain of this moiety. Accordingly, a novel class of amide derivatives (11-25) was synthesized and their SAHase inhibitory activity were estimated. These compounds were also designed to examine the role of different substitutes at aryl (Ar) position of phenylazanediyl diacetic acid and different substitutes in the side chain. It is hoped that continued research will lead to the development of new lead compounds from $N$-(carbamoylmethyl)glycinamide derivatives as effective SAHase inhibitors.

\section{Results and Discussion}

Chemistry The general method for the synthesis of the amide derivatives 11-25 was described in Chart 1. Bromination of the compound 1 with $N$-bromosuccinimide (NBS) gave intermediate 2 in $80 \%$ yield. ${ }^{14,15)}$ Treatment of 2 with $N$-tertbutoxycarbonyl- $N$-methylhydrazine to afford compound $\mathbf{3}$ in $75 \%$ yield. ${ }^{13)}$ Finally, the deprotection of tert-butoxycarbonyl (Boc) group on amino group by hydrolysis with concentrated
Fig. 1. Chemical Structures of Some of the SAHase Inhibitors<smiles>Nc1ncnc2c1ncn2[C@@H]1O[C@H](CO)[C@@H](O)[C@H]1O</smiles><smiles>COC(=O)C(O)CCn1cnc2c(N)ncnc21</smiles>

DZ2002 


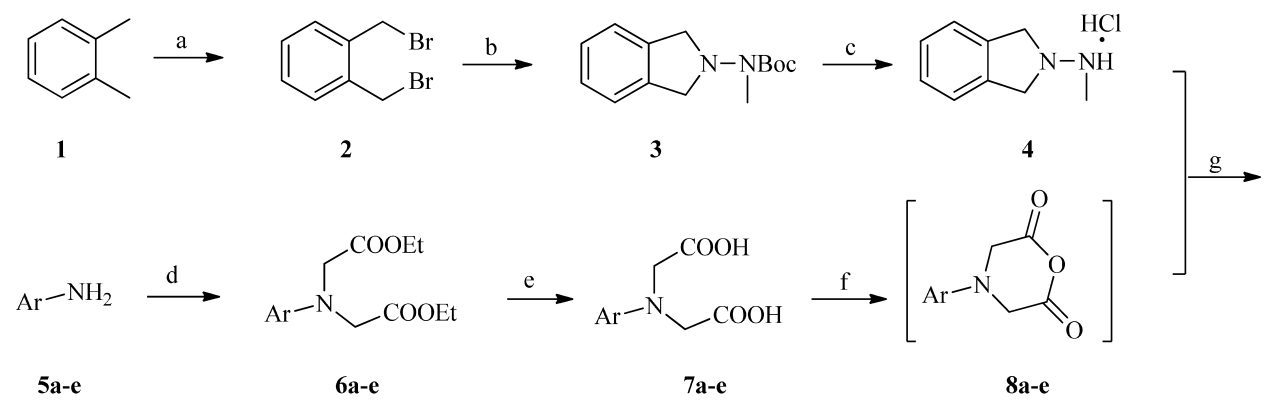

$\mathrm{Ar}=$ (a) 1-methyl-1 $H$-indazol-5-yl; (b) 2-methyl-2 $H$-indazol-5-yl;

(c) 4-(1H-pyrrol-1-yl)phenyl; (d) 4-fluorophenyl; (e) 4-methylphenyl<smiles>Cn1ncc2cc(I)ccc21</smiles>

(a)<smiles></smiles>

(b)<smiles>Cc1ccc(-n2cccc2)cc1</smiles>

(c)<smiles>Cc1ccc(I)cc1</smiles>

(d) (e)<smiles></smiles>

11: $\mathrm{Ar}=1$-methyl- $1 H$-indazol-5-yl, $\mathrm{NR}_{1} \mathrm{R}_{2}=$ ethylamino 12: $\mathrm{Ar}=1$-methyl- $1 H$-indazol-5-yl, $\mathrm{NR}_{1} \mathrm{R}_{2}=$ piperidinyl 13: $\mathrm{Ar}=1$-methyl- $1 H$-indazol-5-yl, $\mathrm{NR}_{1} \mathrm{R}_{2}=$ diethylamino 14: $\mathrm{Ar}=2$-methyl- $2 H$-indazol-5-yl, $\mathrm{NR}_{1} \mathrm{R}_{2}=$ ethylamino 15: $\mathrm{Ar}=2$-methyl- $2 H$-indazol-5-yl, $\mathrm{NR}_{1} \mathrm{R}_{2}=$ piperidinyl 16: $\mathrm{Ar}=2$-methyl- $2 H$-indazol-5-yl, $\mathrm{NR}_{1} \mathrm{R}_{2}=$ diethylamino 17: $\mathrm{Ar}=4-\left(1 H\right.$-pyrrol-1-yl)phenyl, $\mathrm{NR}_{1} \mathrm{R}_{2}=$ ethylamino 18: $\mathrm{Ar}=4-\left(1 H\right.$-pyrrol-1-yl)phenyl, $\mathrm{NR}_{1} \mathrm{R}_{2}=$ piperidinyl 19: $\mathrm{Ar}=4-\left(1 H\right.$-pyrrol-1-yl)phenyl, $\mathrm{NR}_{1} \mathrm{R}_{2}=$ diethylamino
20: $A r=4$-fluorophenyl, $\mathrm{NR}_{1} \mathrm{R}_{2}=$ ethylamino 21: $\mathrm{Ar}=4$-fluorophenyl, $\mathrm{NR}_{1} \mathrm{R}_{2}=$ piperidinyl 22: $\mathrm{Ar}=4$-fluorophenyl, $\mathrm{NR}_{1} \mathrm{R}_{2}=$ diethylamino 23: $\mathrm{Ar}=4$-methylphenyl, $\mathrm{NR}_{1} \mathrm{R}_{2}=$ ethylamino 24: $\mathrm{Ar}=4$-methylphenyl, $\mathrm{NR}_{1} \mathrm{R}_{2}=$ piperidinyl 25: $\mathrm{Ar}=4$-methylphenyl, $\mathrm{NR}_{1} \mathrm{R}_{2}=$ diethylamino

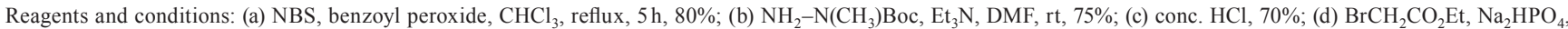
$\mathrm{NaI}, \mathrm{CH}_{3} \mathrm{CN}$, reflux, $12 \mathrm{~h}$; (e) $\mathrm{KOH}$, EtOH then $2 \mathrm{~N} \mathrm{HCl}, 35-45 \%$ in 2 steps; (f) $\mathrm{Ac}_{2} \mathrm{O}, 90^{\circ} \mathrm{C}, 3 \mathrm{~h}$; (g) Et ${ }_{3} \mathrm{~N}$, THF, rt; (h) EDCI, HOBt, DMF, Et ${ }_{3} \mathrm{~N}$, rt, $34-47 \%$ in 3 steps.

Chart 1. Synthetic Protocol of the Amide Derivatives 11-25

hydrochloric acid was carried out at room temperature then transformed to compound $\mathbf{4}$ as hydrochloride salt in $70 \%$ yield. ${ }^{16)}$ The corresponding aromatic amines $\mathbf{5 a}-\mathbf{e}$ were firstly alkylated with ethyl bromoacetate under the basic condition to give compounds $\mathbf{6 a}-\mathbf{e},{ }^{17)}$ which were hydrolyzed with potassium hydroxide in ethanol afforded the desired substituted phenylazanediyl diacetic acids $7 \mathbf{a}-\mathbf{e}$. The intermediates $\mathbf{8 a}-\mathbf{e}$ were obtained by dehydration of the substituted phenylazanediyl diacetic acids $7 \mathbf{a}-\mathbf{e}$ in the presence of acetic anhydride at $90^{\circ} \mathrm{C} .{ }^{18)}$ Treatment of $\mathbf{8 a}-\mathbf{e}$ with $\mathbf{4}$ under the basic condition to give monacid derivatives $9 \mathbf{a}-\mathbf{e}$. The amide derivatives 11-25 were prepared by condensation of monacid derivatives $\mathbf{9 a}-\mathbf{e}$ and various ethylenediamine derivatives 10 with 1-ethyl-(3-(3-dimethylamino)propyl)-carbodiimide hydrochloride (EDCI) as condensation agent and 1-hydroxybenzotriazole (HOBt) as catalyst in $N, N$-dimethylformamide (DMF) in good yield. ${ }^{18,19)}$ The chemical structures of all the synthesized compounds were characterized by ${ }^{1} \mathrm{H}-\mathrm{NMR}$, MS and elemental analysis $(\mathrm{C}, \mathrm{H}, \mathrm{N})$.

Biological Activity All the newly synthesized compounds, along with the reference compounds aristeromycin and 3-DZA, were screened for their SAHase inhibitory activities using the method which descried in literature. ${ }^{20,21)}$ The results, expressed as inhibition ration and $\mathrm{IC}_{50}$ values, were summarized in Table 1.

As shown in Table 1, most of the compounds displayed potent SAHase inhibitory activities. Five compounds (11, 12, 14, 15, 17) displayed more potent SAHase inhibitory activities than the reference inhibitor $3-\mathrm{DZA}\left(\mathrm{IC}_{50}=5.52 \mu \mathrm{M}\right)$. The most promising compound $11\left(\mathrm{IC}_{50}=0.48 \mu \mathrm{M}\right)$ and $14\left(\mathrm{IC}_{50}=0.44 \mu \mathrm{M}\right)$ exhibited more potent SAHase inhibition effects than the reference inhibitor aristeromycin $\left(\mathrm{IC}_{50}=0.49 \mu \mathrm{M}\right)$, one of the most potent SAHase inhibitors known so far. Meanwhile, compounds 13, 18 and 19 also exhibited SAHase inhibitory activities in some extent.

All of the amide derivatives 11-25. In most case, compounds with substituted indazole-5-yl group (11-15) displayed potent SAHase inhibitory activities (except compound 16). Particularly, compound $\mathbf{1 4}$ was found to be the most active compounds of this series. When the 2-methyl-2 $H$-indazole5 -yl group of compound 14 was replaced with 4-(1H-pyrrol1-yl) phenyl group as in compound 17, a slight decrease in 
Table 1. The SAHase Inhibitory Activity of 11-25

\begin{tabular}{crcc}
\hline \hline \multirow{2}{*}{ Compound } & \multicolumn{2}{c}{ Inhibition ration $(\%)$} & \\
\cline { 2 - 3 } $\mathrm{IC}_{50}(\mu \mathrm{M})^{a)}$ \\
\cline { 2 - 3 } $\mathbf{1 1}$ & $0.5 \mu \mathrm{M}$ & $2.5 \mu \mathrm{M}$ & \\
$\mathbf{1 2}$ & $24.24 \pm 1.78$ & $54.24 \pm 1.12$ & 0.48 \\
$\mathbf{1 3}$ & $9.53 \pm 1.70$ & $30.19 \pm 3.02$ & 2.03 \\
$\mathbf{1 4}$ & $69.58 \pm 0.72$ & $86.56 \pm 1.10$ & 0.61 \\
$\mathbf{1 5}$ & $17.24 \pm 1.30$ & $38.24 \pm 1.46$ & 4.13 \\
$\mathbf{1 6}$ & $15.97 \pm 2.35$ & $27.15 \pm 1.24$ & 25.34 \\
$\mathbf{1 7}$ & $49.72 \pm 0.20$ & $79.46 \pm 2.83$ & 0.73 \\
$\mathbf{1 8}$ & $10.92 \pm 0.91$ & $21.46 \pm 3.38$ & 12.12 \\
$\mathbf{1 9}$ & $6.41 \pm 0.23$ & $20.16 \pm 0.84$ & 14.08 \\
$\mathbf{2 0}$ & $4.23 \pm 0.14$ & $8.23 \pm 1.02$ & 78.82 \\
$\mathbf{2 1}$ & $0 \pm 0.32$ & $2.63 \pm 0.41$ & $>500$ \\
$\mathbf{2 2}$ & $0 \pm 0.96$ & $4.56 \pm 0.77$ & $>500$ \\
$\mathbf{2 3}$ & $8.23 \pm 0.92$ & $19.23 \pm 1.35$ & 15.60 \\
$\mathbf{2 4}$ & $4.56 \pm 0.45$ & $6.97 \pm 0.87$ & 101.9 \\
$\mathbf{2 5}$ & $0 \pm 0.43$ & $4.89 \pm 0.45$ & 214 \\
Aristeromycin & $83.93 \pm 0.90$ & $98.35 \pm 0.16$ & $0.49^{b)}$ \\
3 -DZA & $2.16 \pm 0.47$ & $35.37 \pm 3.2$ & $5.52^{c)}$ \\
\hline
\end{tabular}

a) Values were determined from logarithmic concentration-inhibition curves an are given as means of three experiments. b) Values in the literature is $0.2 \mu \mathrm{m}$, the SAHase from Mycobacterium tuberculosis. ${ }^{22)}$ c) Values in the literature is $20 \mu \mathrm{m}$, the SAHase from Mycobacterium tuberculosis ${ }^{22}$

SAHase inhibition potency was observed. However, when the 2-methyl-2H-indazole-5-yl group of compound 14 was replaced with 4-methylphenyl group as in compound $\mathbf{2 3}$, a significant decrease in SAHase inhibition potency was observed. Unfortunately replacement of 2-methyl- $2 H$-indazole5-yl group of compound $\mathbf{1 4}$ with 4-fluorophenyl to afford corresponding compounds $\mathbf{2 0 - 2 2}$ resulted in a loss of activity, and 4-methylphenyl derivatives $\mathbf{2 4}$ and 25 were also devoid of SAHase inhibitory activities. These facts imply that the introduction of substituted indazole-5-yl group on the Ar region obviously affected the SAHase inhibitory activity, and in the present investigation, 4-(1H-pyrrol-1-yl) phenyl group facilitated their inhibitory activities, while 4-fluorophenyl group and 4-methylphenyl group were unfavorable.

Substituted ethylenediamine structure at the side chain also affects the SAHase inhibitory activity. Compounds 11, 14, 17, 20 and 23 bearing ethylamino group at side chain exhibited highest influence on SAHase inhibitory activity, followed by the corresponding compounds with piperidinyl group at side chain $(12,15,18,21,24)$. However, the corresponding compounds with diethylamino group at side chain $(13,16,19,22$, 25) displayed lower SAHase inhibition potency. These results indicate that the ethylamino group is considered to be the best substituent at side chain showing potent SAHase inhibitory activity.

\section{Conclusion}

In summary, a novel class of amide derivatives were designed, synthesized and evaluated as SAHase inhibitors. The results demonstrated that most of target compounds displayed potent SAHase inhibitory activities. Particularly, compounds $\mathbf{1 1}$ and $\mathbf{1 4}$ exhibited more potent inhibitory effects than the aristeromycin and 3-DZA. The compounds with 1-methyl- $1 H$ indazole-5-yl group or 2-methyl-2H-indazole-5-yl group at $\mathrm{Ar}$ position and ethylamino group at side chain showed better SAHase inhibitory activities. These results showed above are very encouraging, and further investigation of this kind of compounds may be of interest.

\section{Experimental}

Chemistry Melting points were determined on WRS-21 melting point apparatus and were uncorrected. ${ }^{1} \mathrm{H}-\mathrm{NMR}$ spectra were recorded on INOVA $400(400 \mathrm{MHz})$ spectrometer with tetramethylsilane (TMS) as an internal standard. Chemical shifts $(\delta)$ are in ppm relative to TMS, and coupling constants $(J)$ are expressed in hertz $(\mathrm{Hz})$. Electron-spray ionization mass spectra (ESI-MS) in positive mode were recorded on a HP5989A mass spectrometer. Column chromatography was performed on 200-300 mesh silica gel. Analytical thin layer chromatography (TLC) was performed on precoated silica gel 60 F254 plates and visualization on TLC was achieved by UV light $(254,354 \mathrm{~nm})$. Unless otherwise stated, all commercial reagents and solvents were used without additional purification.

1,2-Bis(bromomethyl)benzene (2) A mixture of compound 1 (40 g, $0.38 \mathrm{~mol})$, NBS $(140.8 \mathrm{~g}, 0.79 \mathrm{~mol})$, benzoyl peroxide $(0.91 \mathrm{~g}, 3.8 \mathrm{mmol})$ in $\mathrm{CHCl}_{3}(400 \mathrm{~mL})$ was heated for $5 \mathrm{~h}$ under reflux. The reaction mixture was cooled to room temperature and $\mathrm{CH}_{2} \mathrm{Cl}_{2}(400 \mathrm{~mL})$ was added to the mixture. The organic layer was washed with water $(2 \times 200 \mathrm{~mL})$ and dried over $\mathrm{Na}_{2} \mathrm{SO}_{4}$, filtered, and the solvent were removed in vacuo. The residue was recrystallized from $n$-hexane-ethanol $(30: 1,620 \mathrm{~mL})$ to give compound $2(79.0 \mathrm{~g}, 80 \%)$ as white solid, mp $92-93^{\circ} \mathrm{C} .{ }^{1} \mathrm{H}-\mathrm{NMR}\left(400 \mathrm{MHz}, \mathrm{CDCl}_{3}\right) \delta: 4.66(4 \mathrm{H}$, s), 7.29-7.38 (4H, m).

tert-Butyl Isoindolin-2-yl(methyl) Carbamate (3) A mixture of compound 2 (59.7 g, $0.22 \mathrm{~mol})$, tert-butyl methylcarbamate $(34.7 \mathrm{~g}, 0.23 \mathrm{~mol})$ and triethylamine $(44.5 \mathrm{~g}, 0.44 \mathrm{~mol})$ in DMF $(300 \mathrm{~mL})$ was heated for $2 \mathrm{~h}$ at $65^{\circ} \mathrm{C}$. The reaction mixture was cooled to room temperature and water $(400 \mathrm{~mL})$ was added to the mixture, the aqueous phase was extracted with ethyl acetate $(3 \times 300 \mathrm{~mL})$. The combined organic layer was washed with brine and dried over $\mathrm{Na}_{2} \mathrm{SO}_{4}$, filtered, and the solvent were removed in vacuo to get compound $3(35.5 \mathrm{~g}$, $65.0 \%)$ as white solid, mp $62-64^{\circ} \mathrm{C} .{ }^{1} \mathrm{H}-\mathrm{NMR}(400 \mathrm{MHz}$, DMSO- $\left.d_{6}\right) \delta: 1.35(9 \mathrm{H}, \mathrm{s}), 2.99(3 \mathrm{H}, \mathrm{s}), 4.33(4 \mathrm{H}, \mathrm{s}), 7.20-7.22$ $(4 \mathrm{H}, \mathrm{m})$. ESI-MS $m / z: 249.16[\mathrm{M}+\mathrm{H}]^{+}$.

$\mathrm{N}$-Methylisoindolin-2-amine Hydrochloride (4) Compound $3(27.5 \mathrm{~g}, 0.11 \mathrm{~mol})$ was dissolved in concentrated hydrochloric acid $(83 \mathrm{~mL})$, and stirred at room temperature for $12 \mathrm{~h}$. The reaction mixture was evaporated under reduced pressure and co-evaporated with ethanol $(1000 \mathrm{~mL})$. The residue was recrystallized from ethanol $(100 \mathrm{~mL})$ to give compound $4(14.2 \mathrm{~g}, 70 \%)$ as white solid, mp $160-162^{\circ} \mathrm{C}$. ${ }^{1} \mathrm{H}-\mathrm{NMR}$ $\left(400 \mathrm{MHz}, \mathrm{DMSO}-d_{6}+\mathrm{D}_{2} \mathrm{O}\right) \delta: 2.88(3 \mathrm{H}, \mathrm{s}), 4.59(4 \mathrm{H}, \mathrm{s})$, 7.41-7.42 (4H, m). ESI-MS m/z: $149.11[\mathrm{M}+\mathrm{H}]^{+}$.

General Procedure for the Synthesis of Substituted Phenylazanediyl Diacetic Acids (7a-e) A mixture of aromatic amines 5a-e $(0.1 \mathrm{~mol})$, ethyl 2-bromoacetate $(0.21 \mathrm{~mol})$, $\mathrm{Na}_{2} \mathrm{HPO}_{4}(0.25 \mathrm{~mol})$ and $\mathrm{NaI}(0.05 \mathrm{~mol})$ in $\mathrm{CH}_{3} \mathrm{CN}(250 \mathrm{~mL})$ was heated for $12 \mathrm{~h}$ under reflux. The reaction mixture was cooled to room temperature and concentrated in vacuo, and the water was added to the residue, the aqueous phase was extracted with ethyl acetate. The combined organic layer was washed with brine and dried over $\mathrm{Na}_{2} \mathrm{SO}_{4}$, filtered, and the solvent were removed in vacuo to obtain $\mathbf{6 a}-\mathbf{e}$. The solution of $\mathrm{KOH}(0.25 \mathrm{~mol})$ in ethanol $(500 \mathrm{~mL})$ was added to the so- 
lution of $\mathbf{6 a}-\mathbf{e}$ in ethanol $(100 \mathrm{~mL})$, and the reaction mixture was heated at $60^{\circ} \mathrm{C}$ for $1 \mathrm{~h}$. The reaction mixture was cooled to room temperature, filtered, and the filtrate was dissolved in water. The $\mathrm{pH}$ of the suspension was adjusted to 2 with $2 \mathrm{~N}$ $\mathrm{HCl}$. The crystalline product was precipitated, filtrated and dried under vacuum to give $7 \mathbf{a}-\mathbf{e}$.

2,2'-((1-Methyl-1H-indazol-5-yl)azanediyl)diacetic Acid (7a): Yield 40\% (2 steps), white solid, mp $172-174^{\circ} \mathrm{C}$. ${ }^{1} \mathrm{H}-\mathrm{NMR}$ $\left(400 \mathrm{MHz}, \mathrm{DMSO}-d_{6}\right) \delta: 3.95(3 \mathrm{H}, \mathrm{s}), 4.14(4 \mathrm{H}, \mathrm{s}), 6.87-6.90$ $(1 \mathrm{H}, \mathrm{m}), 7.47(1 \mathrm{H}, \mathrm{d}, J=9.2 \mathrm{~Hz}), 7.81(1 \mathrm{H}, \mathrm{s})$. ESI-MS $m / z$ : $264.12[\mathrm{M}+\mathrm{H}]^{+}$.

2,2'-((2-Methyl-2H-indazol-5-yl)azanediyl)diacetic Acid (7b): Yield 35\% (2 steps), white solid, mp $156-158^{\circ} \mathrm{C}$. ${ }^{1} \mathrm{H}-\mathrm{NMR}$ $\left(400 \mathrm{MHz}, \mathrm{DMSO}-d_{6}\right) \delta: 3.91(3 \mathrm{H}, \mathrm{s}), 4.12(4 \mathrm{H}, \mathrm{s}), 6.70(1 \mathrm{H}$, s), 6.85-6.88 (1H, m), 7.42-7.45 (1H, d, J=9.2 Hz), $7.79(1 \mathrm{H}, \mathrm{s})$. ESI-MS $m / z: 264.16[\mathrm{M}+\mathrm{H}]^{+}$.

2,2'-((4-(1H-Pyrrol-1-yl)phenyl)azanediyl)diacetic Acid (7c): Yield $30 \%$ (2 steps), brown solid, mp $150-152^{\circ} \mathrm{C}$. ${ }^{1} \mathrm{H}-\mathrm{NMR}$ $\left(400 \mathrm{MHz}\right.$ DMSO- $\left.d_{6}+\mathrm{D}_{2} \mathrm{O}\right) \delta: 4.13(4 \mathrm{H}, \mathrm{s}), 6.17-6.20(2 \mathrm{H}, \mathrm{m})$, 6.59-6.66 (2H, m), 7.10-7.26 (2H, m), $7.33(2 \mathrm{H}, \mathrm{d}, J=8.8 \mathrm{~Hz})$. ESI-MS $m / z: 275.12[\mathrm{M}+\mathrm{H}]^{+}$.

2,2'-((4-Fluorophenyl)azanediyl)diacetic Acid (7d): Yield $42 \%$ (2 steps), white solid, mp $192-194^{\circ} \mathrm{C} .{ }^{1} \mathrm{H}-\mathrm{NMR}(400 \mathrm{MHz}$, $\left.\mathrm{CDCl}_{3}\right) \delta: 4.12(4 \mathrm{H}, \mathrm{s}), 6.55-6.57(2 \mathrm{H}, \mathrm{m}), 6.96-7.01(2 \mathrm{H}, \mathrm{m})$. ESI-MS $m / z: 228.06[\mathrm{M}+\mathrm{H}]^{+}$.

2,2'-( $p$-Tolylazanediyl)diacetic Acid (7e): Yield 45\% (2 steps), white solid, mp $202-204^{\circ} \mathrm{C} .{ }^{1} \mathrm{H}-\mathrm{NMR} \quad(400 \mathrm{MHz}$, DMSO- $\left.d_{6}\right) \delta: 2.16(3 \mathrm{H}, \mathrm{s}), 3.99(4 \mathrm{H}, \mathrm{s}), 6.37(2 \mathrm{H}, \mathrm{d}, J=8.8 \mathrm{~Hz})$, $6.97(2 \mathrm{H}, \mathrm{d}, J=8.8 \mathrm{~Hz})$. ESI-MS $m / z: 224.10[\mathrm{M}+\mathrm{H}]^{+}$.

General Procedure for the Synthesis of Amide Derivatives (11-25) A mixture of substituted phenylazanediyl diacetic acids $7 \mathbf{a}-\mathbf{e}(3.8 \mathrm{mmol})$ and acetic anhydride $(20 \mathrm{~mL})$ was heated to $90^{\circ} \mathrm{C}$ for $3 \mathrm{~h}$, and the reaction mixture was concentrated in vacuo to get intermediates $\mathbf{8 a}-\mathbf{e}$. A solution of triethylamine $(9.5 \mathrm{mmol})$ in tetrahydrofuran (THF) $(20 \mathrm{~mL})$ was added to the corresponding intermediates $\mathbf{8 a}-\mathbf{e}$, the mixture was stirred at room temperature for $10 \mathrm{~min}$. Compound $4(3.8 \mathrm{mmol})$ was added in three portions and the resulting mixture was stirred at room temperature for $12 \mathrm{~h}$. The solution was concentrated and water was added to the residue. The mixture was extracted with ethyl acetate-THF $(10: 1)$ and the combined organic layer was dried over $\mathrm{Na}_{2} \mathrm{SO}_{4}$, filtered, then evaporated to remove solvent to obtain corresponding monacid derivatives $\mathbf{9 a}-\mathbf{e}$. To a stirred solution of $\mathbf{9 a}-\mathbf{e}$ in $\mathrm{DMF}$ $(20 \mathrm{~mL})$ was added triethylamine $(9.5 \mathrm{mmol})$, EDCI $(7.6 \mathrm{mmol})$ and HOBt $(7.6 \mathrm{mmol})$. After the addition was completed, the reaction mixture was stirred for $10 \mathrm{~min}$ at room temperature. A solution of corresponding ethylenediamine derivatives $\mathbf{1 0}$ $(4.56 \mathrm{mmol})$ was added to the reaction mixture, and the resulting mixture was stirred at room temperature for $12 \mathrm{~h}$. The reaction mixture was poured into water. The $\mathrm{pH}$ of the suspension was adjusted to 10 with $5 \%$ aqueous $\mathrm{NaOH}$, and the aqueous phase was extracted with ethyl acetate. The combined organic layer was washed with brine and dried over $\mathrm{Na}_{2} \mathrm{SO}_{4}$, filtered, evaporated, and purified by flash column chromatography $\left(\mathrm{CHCl}_{3}-\mathrm{CH}_{3} \mathrm{OH}=8: 1\right)$ to afford amide derivatives 11-25.

2-((2-((2-(Ethylamino)ethyl)amino)-2-oxoethyl)(1-methyl-1Hindazol-5-yl)amino)- $N$-(isoindolin-2-yl)- $N$-methylacetamide (11): Yield 39\% (3 steps), white solid, mp $178-179^{\circ} \mathrm{C} .{ }^{1} \mathrm{H}-\mathrm{NMR}$ $\left(400 \mathrm{MHz}, \mathrm{DMSO}-d_{6}\right) \delta: 0.91(3 \mathrm{H}, \mathrm{t}, J=7.2 \mathrm{~Hz}), 2.51-2.61(4 \mathrm{H}$, m), $2.97(3 \mathrm{H}, \mathrm{s}), 3.19-3.22(2 \mathrm{H}, \mathrm{m}), 3.91(3 \mathrm{H}, \mathrm{s}), 3.98(2 \mathrm{H}, \mathrm{s})$, 4.27-4.38 (4H, m), 4.66 (2H, s), 6.60-6.87 (2H, m), 7.24-7.46 $(5 \mathrm{H}, \mathrm{m}), 8.93(1 \mathrm{H}, \mathrm{brs})$. ESI-MS $m / z: 464.27[\mathrm{M}+\mathrm{H}]^{+}$. Anal. Calcd for $\mathrm{C}_{25} \mathrm{H}_{33} \mathrm{~N}_{7} \mathrm{O}_{2}$ : C, 64.77; H, 7.18; N, 21.15. Found: C, 64.87; H, 7.24; N, 21.03.

$N$-(Isoindolin-2-yl)- $N$-methyl-2-((1-methyl-1H-indazol-5yl)(2-oxo-2-((2-(piperidin-1-yl)ethyl)amino)ethyl)amino)acetamide (12): Yield $47 \%$ (3 steps), white solid, mp $124-125^{\circ} \mathrm{C}$. ${ }^{1} \mathrm{H}-\mathrm{NMR} \quad\left(400 \mathrm{MHz}, \quad \mathrm{DMSO}-d_{6}\right) \quad \delta: 1.28-1.39$ $(6 \mathrm{H}, \mathrm{m}), 2.42-2.48(6 \mathrm{H}, \mathrm{m}), 2.98(3 \mathrm{H}, \mathrm{s}), 3.22-3.26(2 \mathrm{H}$, m), 3.95-4.01 (5H, m), 4.37-4.40 (4H, m), $4.65(2 \mathrm{H}, \mathrm{s}), 6.58$ $(1 \mathrm{H}, \mathrm{s}), 6.71-6.74(1 \mathrm{H}, \mathrm{m}), 7.24-7.37(4 \mathrm{H}, \mathrm{m}), 7.46-7.48(1 \mathrm{H}$, m), $7.82(1 \mathrm{H}, \mathrm{s}), 8.89(1 \mathrm{H}, \mathrm{brs})$. ESI-MS m/z: $504.54[\mathrm{M}+$ $\mathrm{H}]^{+}$. Anal. Calcd for $\mathrm{C}_{28} \mathrm{H}_{37} \mathrm{~N}_{7} \mathrm{O}_{2}:$ C, 66.77; H, 7.40; N, 19.47. Found: C, 66.65; H, 7.45; N, 19.56 .

2-((2-((2-(Diethylamino)ethyl)amino)-2-oxoethyl)(1-methyl-1 $H$-indazol-5-yl)amino)- $N$-(isoindolin-2-yl)- $N$ methylacetamide (13): Yield 39.0\% (3 steps), white solid, mp $91-93^{\circ} \mathrm{C}$. ${ }^{1} \mathrm{H}-\mathrm{NMR}\left(400 \mathrm{MHz}, \mathrm{DMSO}-d_{6}\right) \delta: 0.89(6 \mathrm{H}$, $\mathrm{t}, J=6.8 \mathrm{~Hz}), 2.49-2.61(6 \mathrm{H}, \mathrm{m}), 2.96(3 \mathrm{H}, \mathrm{s}), 3.23(2 \mathrm{H}, \mathrm{t}$, $J=6.4 \mathrm{~Hz}), 3.94-3.98(5 \mathrm{H}, \mathrm{m}), 4.36(4 \mathrm{H}, \mathrm{s}), 4.64(2 \mathrm{H}, \mathrm{s}), 6.61$ $(1 \mathrm{H}, \mathrm{s}), 6.74-6.76(1 \mathrm{H}, \mathrm{m}), 7.25-7.33(4 \mathrm{H}, \mathrm{m}), 7.43(1 \mathrm{H}, \mathrm{d}$, $J=9.2 \mathrm{~Hz}), 7.79$ (1H, s), 8.85 (1H, brs). ESI-MS $\mathrm{m} / \mathrm{z}: 492.32$ $[\mathrm{M}+\mathrm{H}]^{+}$. Anal. Calcd for $\mathrm{C}_{27} \mathrm{H}_{37} \mathrm{~N}_{7} \mathrm{O}_{2}: \mathrm{C}, 65.96 ; \mathrm{H}, 7.59 ; \mathrm{N}$, 19.94 . Found: C, 66.04; H, 7.50; N, 19.87.

2-((2-((2-(Ethylamino)ethyl)amino)-2-oxoethyl)(2-methyl- $2 \mathrm{H}$ indazol-5-yl)amino)- $N$-(isoindolin-2-yl)- $N$-methylacetamide (14): Yield 34\% (3 steps), white solid, mp $131-132^{\circ} \mathrm{C} .{ }^{1} \mathrm{H}-\mathrm{NMR}$ $\left(400 \mathrm{MHz}, \mathrm{DMSO}-d_{6}\right) \delta: 0.97(3 \mathrm{H}, \mathrm{t}, J=7.2 \mathrm{~Hz}), 2.68-2.78(4 \mathrm{H}$, m), $2.90(3 \mathrm{H}, \mathrm{s}), 3.27-3.29(2 \mathrm{H}, \mathrm{m}), 3.94(3 \mathrm{H}, \mathrm{s}), 4.00(2 \mathrm{H}$, s), $4.29(4 \mathrm{H}, \mathrm{s}), 4.58(2 \mathrm{H}, \mathrm{s}), 6.61(1 \mathrm{H}, \mathrm{s}), 6.74-6.77(1 \mathrm{H}, \mathrm{m})$, $7.22-7.43(5 \mathrm{H}, \mathrm{m}), 7.78(1 \mathrm{H}, \mathrm{s}), 8.98(1 \mathrm{H}, \mathrm{brs})$. ESI-MS $\mathrm{m} / \mathrm{z}$ : 464.30 $[\mathrm{M}+\mathrm{H}]^{+}$. Anal. Calcd for $\mathrm{C}_{25} \mathrm{H}_{33} \mathrm{~N}_{7} \mathrm{O}_{2}: \mathrm{C}, 64.77 ; \mathrm{H}$, 7.18; N, 21.15; Found: C, 64.69; H, 7.24; N, 21.09.

$N$-(Isoindolin-2-yl)- $N$-methyl-2-((2-methyl- $H$-indazol-5yl)(2-oxo-2-((2-(piperidin-1-yl)ethyl)amino)ethyl)amino)acetamide (15): Yield 40\% (3 steps), white solid, mp $155-157^{\circ} \mathrm{C} .{ }^{1} \mathrm{H}-\mathrm{NMR}\left(400 \mathrm{MHz}, \mathrm{DMSO}-d_{6}\right) \delta$ : $1.25-1.37(6 \mathrm{H}$, m), 2.41-2.51 (6H, m), $2.98(3 \mathrm{H}, \mathrm{s}), 3.25(2 \mathrm{H}, \mathrm{t}, J=6.4 \mathrm{~Hz})$, $3.95-3.98(5 \mathrm{H}, \mathrm{m}), 4.33(4 \mathrm{H}, \mathrm{s}), 4.61(2 \mathrm{H}, \mathrm{s}), 6.61(1 \mathrm{H}, \mathrm{s})$, 6.73-6.76 (1H, m), 7.24-7.45 (5H, m), $7.81(1 \mathrm{H}, \mathrm{s}), 8.85$ $\left(1 \mathrm{H}\right.$, brs). ESI-MS m/z: $504.31[\mathrm{M}+\mathrm{H}]^{+}$. Anal. Calcd for $\mathrm{C}_{28} \mathrm{H}_{37} \mathrm{~N}_{7} \mathrm{O}_{2}$ : C, 66.77; H, 7.40; N, 19.47. Found: C, 66.79; H, $7.34 ; \mathrm{N}, 19.55$.

$2-((2-((2-($ Diethylamino $)$ ethyl $)$ amino $)-2$-oxoethyl $)-$ (2-methyl-2 $H$-indazol-5-yl)amino)- $N$-(isoindolin-2-yl)- $N$ methylacetamide (16): Yield 45\% (3 steps), white solid, mp 98-99 ${ }^{\circ} \mathrm{C} .{ }^{1} \mathrm{H}-\mathrm{NMR}\left(400 \mathrm{MHz}, \mathrm{DMSO}-d_{6}+\mathrm{D}_{2} \mathrm{O}\right) \delta: 0.90(6 \mathrm{H}$, $\mathrm{t}, J=7.2 \mathrm{~Hz}), 2.50-2.62(6 \mathrm{H}, \mathrm{m}), 2.93(3 \mathrm{H}, \mathrm{s}), 3.23(2 \mathrm{H}, \mathrm{t}$, $J=6.4 \mathrm{~Hz}), 3.95-3.99(5 \mathrm{H}, \mathrm{m}), 4.37(4 \mathrm{H}, \mathrm{s}), 4.65$ (2H, s), 6.60 $(1 \mathrm{H}, \mathrm{s}), 6.73(1 \mathrm{H}, \mathrm{d}, J=8.8 \mathrm{~Hz}), 7.23-7.31(4 \mathrm{H}, \mathrm{m}), 7.44(1 \mathrm{H}$, $\mathrm{d}, J=8.8 \mathrm{~Hz}), 7.80(1 \mathrm{H}, \mathrm{s}), 8.85(1 \mathrm{H}, \mathrm{brs})$. ESI-MS $m / z: 492.35$ $[\mathrm{M}+\mathrm{H}]^{+}$. Anal. Calcd for $\mathrm{C}_{27} \mathrm{H}_{37} \mathrm{~N}_{7} \mathrm{O}_{2}: \mathrm{C}, 65.96 ; \mathrm{H}, 7.59 ; \mathrm{N}$, 19.94. Found: C, 65.90; H, 7.64; N, 19.98 .

$2-((4-(1 H$-Pyrrol-1-yl)phenyl)(2-((2-(ethylamino)ethyl)amino)-2-oxoethyl)amino)- $N$-(isoindolin-2-yl)- $N$ methylacetamide (17): Yield 31\% (3 steps), white solid, mp 133-135 ${ }^{\circ} \mathrm{C} .{ }^{1} \mathrm{H}-\mathrm{NMR} \quad\left(400 \mathrm{MHz}\right.$, DMSO- $\left.d_{6}\right) \delta: 1.09(3 \mathrm{H}$, $\mathrm{t}, J=7.2 \mathrm{~Hz}), 2.79-2.87(4 \mathrm{H}, \mathrm{m}), 2.95(3 \mathrm{H}, \mathrm{s}), 3.20(2 \mathrm{H}, \mathrm{t}$, $J=6.4 \mathrm{~Hz}), 3.99(2 \mathrm{H}, \mathrm{s}), 4.34(4 \mathrm{H}, \mathrm{s}), 4.63(2 \mathrm{H}, \mathrm{s}), 6.18-6.19$ $(2 \mathrm{H}, \mathrm{m}), 6.50(2 \mathrm{H}, \mathrm{d}, J=9.2 \mathrm{~Hz}), 7.11-7.13(2 \mathrm{H}, \mathrm{m}), 7.25-7.39$ 
$(6 \mathrm{H}, \mathrm{m}), 8.90\left(1 \mathrm{H}\right.$, brs). ESI-MS m/z: $475.35[\mathrm{M}+\mathrm{H}]^{+}$. Anal. Calcd for $\mathrm{C}_{27} \mathrm{H}_{34} \mathrm{~N}_{6} \mathrm{O}_{2}: \mathrm{C}, 68.33 ; \mathrm{H}, 7.22 ; \mathrm{N}, 17.71$. Found: $\mathrm{C}$, 68.27; H, 7.26; N, 17.77 .

2-((4-(1H-Pyrrol-1-yl)phenyl)(2-oxo-2-((2-(piperidin-1yl)ethyl)amino)ethyl) a mino) - N-(isoindolin-2-yl)- $N$ methylacetamide (18): Yield 39\% (3 steps), white solid, mp $124-126^{\circ} \mathrm{C} .{ }^{1} \mathrm{H}-\mathrm{NMR}\left(400 \mathrm{MHz}, \mathrm{DMSO}-d_{6}\right) \delta: 1.40-1.45(6 \mathrm{H}$, m), 2.49-2.51 (6H, m), $2.98(3 \mathrm{H}, \mathrm{s}), 3.98(2 \mathrm{H}, \mathrm{s}), 4.36(4 \mathrm{H}$, s), $4.65(2 \mathrm{H}, \mathrm{s}), 6.15-6.18(2 \mathrm{H}, \mathrm{m}), 6.48(2 \mathrm{H}, \mathrm{d}, J=9.2 \mathrm{~Hz})$, 7.12-7.13 (2H, m), 7.27-7.35 (6H, m), 8.90 (1H, br s). ESI-MS $m / z$ : $515.33[\mathrm{M}+\mathrm{H}]^{+}$. Anal. Calcd for $\mathrm{C}_{30} \mathrm{H}_{38} \mathrm{~N}_{6} \mathrm{O}_{2}: \mathrm{C}, 70.01 ; \mathrm{H}$, 7.44; N, 16.33. Found: C, 70.08; H, 7.39; N, 16.38 .

2-((4-(1H-Pyrrol-1-yl)phenyl)(2-((2-(diethylamino)ethyl)amino)-2-oxoethyl)amino)- $N$-(isoindolin-2-yl)- $N$ methylacetamide (19): Yield 44\% (3 steps), white solid, mp 70-72 ${ }^{\circ} \mathrm{C}$. ${ }^{1} \mathrm{H}-\mathrm{NMR} \quad\left(400 \mathrm{MHz}\right.$, DMSO- $\left.d_{6}\right) \delta: 1.04(6 \mathrm{H}, \mathrm{t}$, $J=7.2 \mathrm{~Hz}), 2.89-2.94(9 \mathrm{H}, \mathrm{m}), 3.34-3.37$ (2H, m), $3.99(2 \mathrm{H}$, s), $4.32(4 \mathrm{H}, \mathrm{s}), 4.61(2 \mathrm{H}, \mathrm{s}), 6.18-6.19(2 \mathrm{H}, \mathrm{m}), 6.48-6.51$ (2H, m), 7.12-7.14 (2H, m), 7.28-7.32 (6H, m), 8.98 (1H, brs). ESI-MS $m / z$ : $503.32[\mathrm{M}+\mathrm{H}]^{+}$. Anal. Calcd for $\mathrm{C}_{29} \mathrm{H}_{38} \mathrm{~N}_{6} \mathrm{O}_{2}: \mathrm{C}$, 69.29; H, 7.62; N, 16.72. Found: C, 69.34; H, 7.66; N, 16.63 .

$2-((2-((2-($ Diethylamino $)$ ethyl $)$ amino $)-2$-oxoethyl $)(4-$ fluorophenyl)amino)- $N$-(isoindolin-2-yl)- $N$-methylacetamide (20): Yield 51\% (3 steps), white solid, mp $74-76^{\circ} \mathrm{C} .{ }^{1} \mathrm{H}-\mathrm{NMR}$ $\left(400 \mathrm{MHz}, \mathrm{DMSO}-d_{6}\right) \delta: 1.07(3 \mathrm{H}, \mathrm{t}, J=7.2 \mathrm{~Hz}), 2.70-2.77(4 \mathrm{H}$, m), $2.95(3 \mathrm{H}, \mathrm{s}), 3.27-3.302 \mathrm{H}, \mathrm{m}), 3.94(2 \mathrm{H}, \mathrm{s}), 4.34(4 \mathrm{H}, \mathrm{s})$, $4.61(2 \mathrm{H}, \mathrm{s}), 6.40-6.43(2 \mathrm{H}, \mathrm{m}), 6.97-6.99$ (2H, m), 7.25-7.30 $(4 \mathrm{H}, \mathrm{m}), 8.90(1 \mathrm{H}, \mathrm{brs})$. ESI-MS $m / z: 428.20[\mathrm{M}+\mathrm{H}]^{+}$. Anal. Calcd for $\mathrm{C}_{23} \mathrm{H}_{30} \mathrm{FN}_{5} \mathrm{O}_{2}: \mathrm{C}, 64.62 ; \mathrm{H}, 7.07 ; \mathrm{N}, 16.38$. Found: C, 64.56; H, 7.01; N, 16.44 .

2-((4-Fluorophenyl)(2-oxo-2-((2-(piperidin-1-yl)ethyl)amino)ethyl)amino)- $N$-(isoindolin-2-yl)- $N$-methylacetamide (21): Yield 45\% (3 steps), white solid, mp $145-146^{\circ} \mathrm{C} .{ }^{1} \mathrm{H}-\mathrm{NMR}$ $\left(400 \mathrm{MHz}, \mathrm{DMSO}-d_{6}\right) \delta: 1.33-1.46(3 \mathrm{H}, \mathrm{m}), 2.42-2.50(6 \mathrm{H}$, m), $2.95(3 \mathrm{H}, \mathrm{s}), 3.20-3.28(2 \mathrm{H}, \mathrm{m}), 3.90(2 \mathrm{H}, \mathrm{s}), 4.33(4 \mathrm{H}, \mathrm{s})$, $4.57(2 \mathrm{H}, \mathrm{s}), 6.38-6.42(2 \mathrm{H}, \mathrm{m}), 6.97-7.01(2 \mathrm{H}, \mathrm{m}), 7.25-7.31$ $(4 \mathrm{H}, \mathrm{m}), 8.78\left(1 \mathrm{H}\right.$, brs). ESI-MS $m / z: 468.32[\mathrm{M}+\mathrm{H}]^{+}$. Anal. Calcd for $\mathrm{C}_{26} \mathrm{H}_{34} \mathrm{FN}_{5} \mathrm{O}_{2}$ : C, 66.79; H, 7.33; N, 14.98. Found: C, 66.72; H, 7.28; N, 14.91.

2-((2-((2-(Diethylamino)ethyl)amino)-2-oxoethyl)(4fluorophenyl)amino)- $N$-(isoindolin-2-yl)- $N$-methylacetamide (22): Yield 43\% (3 steps), white solid, mp $85-87^{\circ} \mathrm{C} .{ }^{1} \mathrm{H}-\mathrm{NMR}$ $\left(400 \mathrm{MHz}, \mathrm{DMSO}-d_{6}\right) \delta$ : 0.97-1.05 $(6 \mathrm{H}, \mathrm{m}), 2.69-2.74(6 \mathrm{H}, \mathrm{m})$, $2.92(3 \mathrm{H}, \mathrm{s}), 3.25-3.30(2 \mathrm{H}, \mathrm{m}), 3.91(2 \mathrm{H}, \mathrm{s}), 4.33(4 \mathrm{H}, \mathrm{s}), 4.58$ $(2 \mathrm{H}, \mathrm{s}), 6.39-6.44(2 \mathrm{H}, \mathrm{m}), 6.96-7.02(2 \mathrm{H}, \mathrm{m}), 7.24-7.31(4 \mathrm{H}$, m), $8.77\left(1 \mathrm{H}\right.$, brs). ESI-MS m/z: $456.28[\mathrm{M}+\mathrm{H}]^{+}$. Anal. Calcd for $\mathrm{C}_{25} \mathrm{H}_{34} \mathrm{FN}_{5} \mathrm{O}_{2}$ : C, 65.91; H, 7.52; N, 15.37. Found: C, 65.95; $\mathrm{H}, 7.48 ; \mathrm{N}, 15.44$.

2-((2-((2-(Ethylamino)ethyl)amino)-2-oxoethyl)( $p$-tolyl)amino)- $N$-(isoindolin-2-yl)- $N$-methylacetamide (23): Yield $54 \%$ (3 steps), white solid, mp $162-163^{\circ} \mathrm{C} .{ }^{1} \mathrm{H}-\mathrm{NMR}(400 \mathrm{MHz}$, DMSO- $\left.d_{6}\right) \delta: 1.11(3 \mathrm{H}, \mathrm{t}, J=7.2 \mathrm{~Hz}), 2.17(3 \mathrm{H}, \mathrm{m}), 2.73-2.81$ $(4 \mathrm{H}, \mathrm{m}), 2.99(3 \mathrm{H}, \mathrm{s}), 3.32-3.35(2 \mathrm{H}, \mathrm{m}), 3.93(2 \mathrm{H}, \mathrm{s})$, $4.30-4.38$ (4H, m), $4.61(2 \mathrm{H}, \mathrm{s}), 6.32(2 \mathrm{H}, \mathrm{d}, J=8.4 \mathrm{~Hz}), 6.96$ $(2 \mathrm{H}, \mathrm{d}, J=8.4 \mathrm{~Hz}), 7.26-7.33(4 \mathrm{H}, \mathrm{m}), 9.06(1 \mathrm{H}, \mathrm{s})$. ESI-MS $m / z: 424.20[\mathrm{M}+\mathrm{H}]^{+}$. Anal. Calcd for $\mathrm{C}_{24} \mathrm{H}_{33} \mathrm{~N}_{5} \mathrm{O}_{2}: \mathrm{C}, 68.06 ; \mathrm{H}$, 7.85; N, 16.53. Found: C, 68.13; H, 7.79; N, 16.48.

$N$-(Isoindolin-2-yl)- $N$-methyl-2-((2-oxo-2-((2-(piperidin-1yl)ethyl)amino)ethyl)( $p$-tolyl)amino)acetamide (24): Yield $48 \%$ (3 steps), white solid, mp $117-119^{\circ} \mathrm{C} .{ }^{1} \mathrm{H}-\mathrm{NMR}(400 \mathrm{MHz}$, DMSO- $\left.d_{6}\right) \delta: 1.23-1.42(6 \mathrm{H}, \mathrm{m}), 2.16(3 \mathrm{H}, \mathrm{s}), 2.26-2.32(6 \mathrm{H}$, m), $2.96(3 \mathrm{H}, \mathrm{s}), 3.16-3.26(2 \mathrm{H}, \mathrm{m}), 3.88(2 \mathrm{H}, \mathrm{s}), 4.28-4.37$ $(4 \mathrm{H}, \mathrm{m}), 4.56(2 \mathrm{H}, \mathrm{s}), 6.31(2 \mathrm{H}, \mathrm{d}, J=8.4 \mathrm{~Hz}), 6.96(2 \mathrm{H}, \mathrm{d}$, $J=8.4 \mathrm{~Hz}), 7.25-7.33(4 \mathrm{H}, \mathrm{m}), 8.84(1 \mathrm{H}, \mathrm{brs})$. ESI-MS $\mathrm{m} / \mathrm{z}$ : 464.19 $[\mathrm{M}+\mathrm{H}]^{+}$. Anal. Calcd for $\mathrm{C}_{27} \mathrm{H}_{37} \mathrm{~N}_{5} \mathrm{O}_{2}: \mathrm{C}$, 69.95; $\mathrm{H}$, 8.04; N, 15.11. Found: C, 70.01; H, 8.09; N, 15.03.

2-((2-((2-(Diethylamino)ethyl)amino)-2-oxoethyl)( $p$-tolyl)amino)- $N$-(isoindolin-2-yl)- $N$-methylacetamide (25): Yield $46 \%$ (3 steps), white solid, mp $137-138^{\circ} \mathrm{C} .{ }^{1} \mathrm{H}-\mathrm{NMR}(400 \mathrm{MHz}$, DMSO- $\left.d_{6}\right) \delta: 0.96(6 \mathrm{H}, \mathrm{t}, J=7.2 \mathrm{~Hz}), 2.16(3 \mathrm{H}, \mathrm{s}), 2.54-2.61$ $(6 \mathrm{H}, \mathrm{m}), 2.95(3 \mathrm{H}, \mathrm{s}), 3.23(2 \mathrm{H}, \mathrm{m}), 3.89(2 \mathrm{H}, \mathrm{s}), 4.29-4.37$ $(4 \mathrm{H}, \mathrm{m}), 4.57(2 \mathrm{H}, \mathrm{s}), 6.31(2 \mathrm{H}, \mathrm{d}, J=8.4 \mathrm{~Hz}), 6.95(2 \mathrm{H}, \mathrm{d}$, $J=8.4 \mathrm{~Hz}), 7.25-7.33(4 \mathrm{H}, \mathrm{m}), 8.87(1 \mathrm{H}, \mathrm{brs})$. ESI-MS $\mathrm{m} / \mathrm{z}$ : $452.26[\mathrm{M}+\mathrm{H}]^{+}$. Anal. Calcd for $\mathrm{C}_{26} \mathrm{H}_{37} \mathrm{~N}_{5} \mathrm{O}_{2}: \mathrm{C}, 69.15 ; \mathrm{H}$, 8.26; N, 15.51. Found: C, 69.09; H, 8.29; N, 15.57.

SAHase Inhibition Assay The activity was measured following the procedure described previously with a slight modification. ${ }^{19,20)}$ This method involves the hydrolytic conversion of SAH into ADO and HCY. SAHase from human placenta, SAH was obtained from Sigma, adenosine deaminase (ADA) and ThioGlo 1 were obtained from Calbiochem. SAHase and all compounds were cultured in phosphate buffer ( $\mathrm{pH}$ 8.0) before initiated the reaction. ThioGlo 1 solution was freshly prepared in phosphate buffer $(\mathrm{pH} 8.0)$ before initiated the reaction. The reaction mixture $(50 \mu \mathrm{L})$ contained ethylenediaminetetraacetic acid (50 mM), ADA (0.03 U), SAH $(1.68 \mu \mathrm{M})$, SAHase $(0.5 \mathrm{mU})$ and various concentrations of inhibitors. The reaction took place at $37^{\circ} \mathrm{C}$ for $10 \mathrm{~min}$, and the reaction mixture was quenched through the addition of $50 \mu \mathrm{L}$ pure icecold isopropanol. A solution of $100 \mu \mathrm{L}$ of $20 \mu \mathrm{M}$ ThioGlol in dimethyl sulfoxide was added to reaction mixture. The plate was maintained in the dark at room temperature for $10 \mathrm{~min}$, and the fluorescence was read using the SpectraMax M5 spectrophotometer with $380 \mathrm{~nm}$ excitation and $510 \mathrm{~nm}$ emission filters. One unit of inhibitory activity was defined as the amount of the sample needed to inhibit one unit of enzyme activity. $\mathrm{IC}_{50}$ value was determined by lineal regression of the dose-response curves and was defines as the amount of the sample needed to inhibit the $50 \%$ of control enzyme activity.

Acknowledgment The work was supported by 'New Drug Innovation 2009X09313-006' from the Ministry of Science and Technology of China.

\section{References}

1) Palmer J. L., Abeles R. H., J. Biol. Chem., 251, 5817-5819 (1976).

2) Ueland P. M., Pharmacol. Rev., 34, 223-253 (1982).

3) Palmer J. L., Abeles R. H., J. Biol. Chem., 254, 1217-1226 (1979).

4) De Clercq E., Biochem. Pharmacol., 36, 2567-2575 (1987).

5) Wolfe M. S., Borchardt R. T., J. Med. Chem., 34, 1521-1530 (1991).

6) Henderson D. M., Hanson S., Allen T., Wilson K., Coulter-Karis D. E., Greenberg M. L., Hershfield M. S., Ullman B., Mol. Biochem. Parasitol., 53, 169-183 (1992).

7) Hayden A., Johnson P. W. M., Packham G., Crabb S. J., Breast Cancer Res. Treat., 127, 109-119 (2011).

8) Wu Q. L., Fu Y. F., Zhou W. L., Wang J. X., Feng Y. H., Liu J., Xu J. Y., He P. L., Zhou R., Tang W., Wang G. F., Zhou Y., Yang Y. F., Ding J., Li X. Y., Chen R. X., Yuan C., Lawson B. R., Zuo J. P., J. Pharmacol. Exp. Ther., 313, 705-711 (2005).

9) Zhang Y. M., Ding Y., Tang W., Luo W., Gu M., Lu W., Tang J., Zuo J. P., Nan F. J., Bioorg. Med. Chem., 16, 9212-9216 (2008).

10) Yuan C. S., Saso Y., Lazarides E., Borchardt R. T., Robins M. J., Expert Opin. Ther. Pat., 9, 1197-1206 (1999). 
11) Langheinrich A. C., Braun-Dullaeus R. C., Walker G., Jeide I., Schilling R., Tammoscheit K., Dreyer T., Fink L., Bohle R. M., Haberbosch W., Atherosclerosis, 171, 181-192 (2003).

12) Steere J. A., Honek J. F., Bioorg. Med. Chem., 11, 3229-3236 (2003).

13) Nakao A., Suzuki H., Tatsumi R., Seki M., Tanaka M., Setsuta T., Iwasaki H., WO 2009125853 (2009).

14) Kreher R. P., Kalischko J., Chem. Ber., 124, 645-654 (1991).

15) Paul V., Sudalai A., Daniel T., Srinivasan K. V., Tetrahedron Lett., 35, 7055-7056 (1994).

16) Carpino L. A., J. Am. Chem. Soc., 79, 4427-4429 (1957).

17) Parkesh R., Clive Lee V., Gunnlaugsson T., Org. Biomol. Chem., 5, 310-317 (2007).
18) Yoshida K. I., Nakayama K., Kuru N., Kobayashi S., Ohtsuka M., Takemura M., Hoshino K., Kanda H., Zhang J. Z., Lee V. J., Watkins W. J., Bioorg. Med. Chem., 14, 1993-2004 (2006).

19) Huang X., Zhu J., Broadbent S., Tetrahedron Lett., 51, 1554-1557 (2010).

20) Lozada-Ramírez J. D., Martínez-Martínez I., Sánchez-Ferrer A., García-Carmona F., J. Biochem. Biophys. Methods, 67, 131-140 (2006).

21) Hudec R., Hamada K., Mikoshiba K., Anal. Biochem., 433, 95-101 (2013).

22) Reddy M. C. M., Kuppan G., Shetty N. D., Owen J. L., Ioerger T. R., Sacchettini J. C., Protein Sci., 17, 2134-2144 (2008). 